\title{
Ultrafast All-Optical Wavelength Conversion in Silicon Waveguides using Femtosecond Pump-Probe Pulses.
}

R. Dekker (1), J. Niehusmann (2), M. Först (2), T. Wahlbrink (3), C. Moormann(3) and A. Driessen (1). 1 : Integrated Optical Micro Systems, Mesa+ Research Institute, University of Twente, P.O. Box 217, 7500 AE Enschede, The Netherlands. E-mail: r.dekker@utwente.nl.

2 : RWTH Aachen University, Institut für Halbleitertechnik, 52074 Aachen, Germany. 3: Advanced Microelectronic Center Aachen (AMICA), AMO GmbH, Huyskensweg 25, 52074 Aachen, Germany.

\begin{abstract}
Experimental results on ultrafast all-optical wavelength conversion in Silicon-on-Insulator waveguides are presented. Red and blue shifts of 10nm have been observed in femtosecond pump-probe experiments. Alloptical switching and the importance of waveguide dispersion are discussed.
\end{abstract}

\section{Introduction}

Silicon photonics is receiving attention increasingly over the last couple of years, especially because of its potential for integration with microelectronics. Substantial progress has been achieved in the field of Raman amplification [1] and other nonlinear effects like two-photon absorption (TPA), self-phase modulation (SPM) [2] and the Kerr effect [3] have been successfully demonstrated and thoroughly investigated. In this paper, we present experimental results of femtosecond pump-probe experiments in Silicon-on-Insulator (SOI) waveguides. Large Kerrinduced wavelength shifts on the probe signal have been observed, whereas the free carrier contribution did not substantially contribute. The importance of waveguide dispersion on the conversion efficiency will be discussed and a microring resonator based device is proposed that exploits the observed wavelength shift for sub-picosecond all-optical switching.

\section{Experimental setup}

Figure 1 shows a schematic representation of our experimental setup. Both the pump $(1554 \mathrm{~nm})$ and probe $(1683 \mathrm{~nm})$ pulses with a FWHM pulse duration of 300 fs are generated using an optical parametric oscillator (OPO), while their beam shapes are being modified by optical beam formers (OBF) in order to optimize the fiber coupling efficiency. The time delay between pump and probe pulses is controlled with an optical delay line (ODL) with 6 femtosecond accuracy. Both beams are combined using a beam splitter (BS), polarized (Pol) and coupled into a $10 \mathrm{~cm}$ piece of polarization maintaining fiber (PMF) using a microscope objective (MO). The output of the PMF is used to couple TM polarized light pulses into our SOI waveguides that have a $450 \mathrm{~nm} \times 300 \mathrm{~nm}(\mathrm{w} \times \mathrm{h})$ cross section (by design) and a length of $7 \mathrm{~mm}$. The transmitted pulses are coupled out using a single mode fiber, which is attached to an optical spectrum analyzer (OSA). Folding mirrors (FM) are used to tap the pump and probe signals to detect their power levels using an optical detector (OD). The pump intensity can be controlled using a neutral density filter (FW).

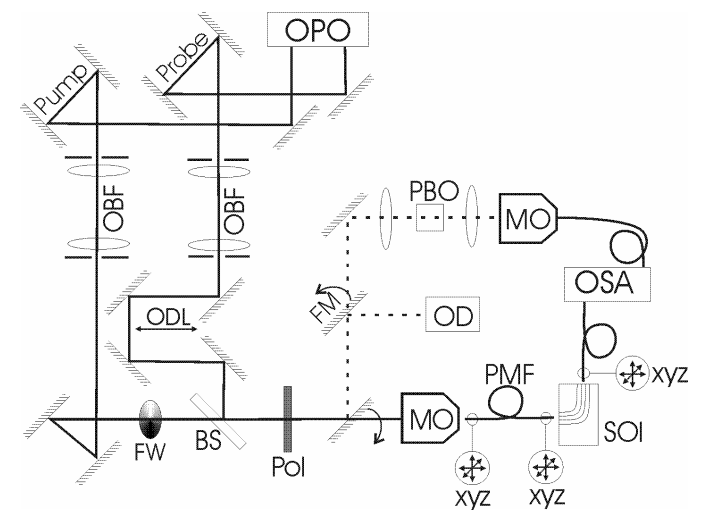

Figure 1: Experimental setup.

\section{Experimental results}

When the strong pump pulses $\left(I_{\max } \sim 10 \mathrm{TW} / \mathrm{m}^{2}\right)$ propagate through the waveguide, the refractive index of the material is changed mainly by two nonlinear effects. The first one is the instantaneous Kerr effect, inducing a refractive index increase of $\Delta n_{K e r r}=n_{2} \cdot l$ [3]. Secondly, by TPA free carriers (FCs) are generated that cause a refractive index decrease of $\Delta n_{F C}=$ $-\left(8.8 \cdot 10^{-22} N+8.5 \cdot 10^{-18} N^{0.8}\right)$ [4], with $N$ the free carrier density. Since both refractive index changes are intensity dependent, they both change with the envelope of the pulse and induce a temporal phase shift $\Delta \phi(t)=2 \pi L \Delta n(t) / \lambda$. Here, $L$ is the interaction length of pump and probe pulses. Consequently, this temporal phase shift causes a frequency shift $\Delta \omega(t)=d \Delta \phi(t) / d t$, that is the basis for SPM and cross phase modulation (XPM). In this paper we will further focus on the pump induced XPM of the probe pulses.



Figure 2: Center wavelength vs. delay time. 
In Figure 2 the experimentally observed center wavelength of the probe pulses are plotted as function of delay time. Here a negative delay time means that the probe pulses are running ahead of the pump pulses, i.e. are overlapping with the leading edge of the pump pulse. We observed a $10 \mathrm{~nm}$ redshift at negative delay times, indicating that the nonlinearities are dominated by the Kerr effect in case of femtosecond pulses. This is in contrast to the blueshift that is normally observed in pump-probe experiments with longer ps pulses [1], where the free carrier dispersion effect is dominant due to the fact that ps pulses are long enough to build up a sufficient amount of free carriers.

\section{Waveguide dispersion}

When the SOI waveguide dimensions are in the submicron range, the geometry induced waveguide dispersion will dominate the material dispersion [5]. The characteristic walkoff length $L_{w}$ represents the distance over which the pump and probe pulses pass through each other's envelope [5]:

$$
L_{w}=T_{0} /\left|\beta_{1 p}-\beta_{1 s}\right|
$$

where $T_{0}$ is the half width of the pulse, and $\beta_{1 p}$ and $\beta_{1 s}$ are the first order dispersion coefficients of the pump and probe, respectively. In Figure 3 the dispersion coefficients and walkoff lengths between the $1554 \mathrm{~nm}$ pump and $1683 \mathrm{~nm}$ probe pulses ( $T_{0}=300 \mathrm{fs}$ ) are plotted for a SOI waveguide with a height of $300 \mathrm{~nm}$ and the width as parameter. It can be seen that the waveguide width is a critical parameter that strongly affects the pump-probe interaction and therefore the maximum attainable wavelength conversion as the conversion efficiency increases with $L_{w}$.



Figure 3: First order dispersion as a function of wavelength and walkoff length vs. waveguide width.

Since the walkoff length scales linearly with the pulse length (Eq. 1), it can be concluded that the interaction length can easily be increased by increasing the pulse lengths. However, at longer pulse lengths the pulse envelope is less steep resulting in weaker wavelength conversion. Therefore we believe that the observed large wavelength shifts in SOI can only be achieved with sub-picosecond pulses, combined with precise control of the waveguide dispersion.

\section{Ultrafast all-optical switching}

Sub-picosecond all-optical switching can be achieved in case that the frequency shifted probe pulse is redirected by a wavelength selective device. In our case we propose a SOI waveguide combined with a SOI microring resonator filter (see Figure 4). The wavelength conversion of $\pm 10 \mathrm{~nm}$ is in the order of the free spectral



Figure 4: Example of a SOI Ring resonator. range (FSR) of a ring resonator with a radius of $10 \mu \mathrm{m}$. This means that the probe signal can be tuned over the full FSR of the ring by adjusting the time delay. Low $Q$ resonators having short loading and unloading times can be used, because of the large pump induced wavelength shift. Since the wavelength conversion is determined by the time derivative of the Kerr induced refractive index change, the switching time of such a switch is related to the width of the pump pulse [1], which is in the order of $300 \mathrm{fs}$. This is in contrast to the all-optical switching mechanisms that rely on the FC dispersion, where the switching speed is limited by the FC lifetime in the order of ns.

\section{Conclusions}

We have shown that both $10 \mathrm{~nm}$ blue and red shifts in SOI waveguides using a pump-probe setup with 300 fs pulses are feasible. The XPM is dominated by the Kerr effect, since the pulses in the femtosecond regime are too short to accumulate a sufficient amount of free carriers to have considerable free carrier dispersion. This means that the temporal refractive index changes are mainly caused by the instantaneous Kerr effect and thus both the wavelength up and down-conversion takes place in a sub-picosecond timeframe. We have shown the importance of waveguide dispersion management on the wavelength conversion efficiency in femtosecond pump-probe experiments. Furthermore, a microring resonator based device is proposed that exploits the observed wavelength shift for sub-picosecond alloptical switching.

\section{References}

[1] Q. Xu et al., Opt.Express, Vol 12 (19), pp 44374442, 2004.

[2] R. Dekker et al., Symposium IEEE/LEOS Benelux Chapter, ISBN 2-9600226-4-5, pp 197-200, 2005.

[3] M. Dinu et al., Appl.Phys.Lett., Vol 82 (18), pp 2954-2956, 2003.

[4] R. A. Soref et al., IEEE.J.Sel.Top.Quant.Electr., Vol QE-23 (1), pp 123-129, 1987.

[5] X. Chen et al., IEEE J. Quant. Electr., Vol 42 (2), pp 160-170, 2006. 